\title{
DIAGNÓSTICOS EN TECNOLOGÍAS DE INFORMACIÓN PARA EL LOGRO DE LOS OBJETIVOS ESTRATÉGICOS EN LAS ORGANIZACIONES
}

\author{
Diagnostics in information technologies for the achievement \\ of strategic objectives in organizations
}

\section{EPISTEMUS}

ISSN: 2007-8196 (electrónico)

ISSN: 2007-4530 (impresa)

Suleika Remedio Frometa ${ }^{1}$

Surayne Torres López ${ }^{2}$

Julio César Espronceda Pérez ${ }^{3}$

Recibido: 15 de septiembre de 2018 ,

Aceptado: 29 de noviembre de 2018

Autor de Correspondencia:

Suleika Remedio Frometa 1

Correo: sremedio@uci.cu

\section{Resumen}

Los diagnósticos tecnológicos son un campo que se enfoca en aconsejar a otras organizaciones como usar las tecnologías de información para conseguir sus objetivos estratégicos. A través del servicio de diagnóstico en tecnologías de la información, se pretende detectar las necesidades y oportunidades de una organización. Realizar un análisis de las diferentes capas o dimensiones de la Arquitectura Empresarial: Negocio, Datos, Aplicaciones, Infraestructura Tecnológica, Recursos Humanos e Integración; las cuales se ven evidenciadas en tres etapas: Inicio, Diagnóstico y Proyección, cada una de ellas realiza actividades encaminadas a mejorar el buen funcionamiento de la organización. El diagnóstico permite dejar de invertir mucho dinero y tiempo en tecnología sin obtener los resultados deseados. A través de metodologías especializadas como la Arquitectura Empresarial, se resolverán problemas y se aprovecharán las oportunidades presentes en el negocio, apoyándose en las tecnologías de la información. La organización obtendrá una alineación entre las metas del negocio y las estrategias de Tecnologías de la Información.

Palabras clave: diagnóstico tecnológico, arquitectura empresarial, tecnologías de información, objetivos estratégicos.

\section{Abstract}

Technologicaldiagnoses are afield that focuseson advising otherorganizations how to use information technologies to achieve their strategic objectives. Through the diagnostic service in information technologies, it is intended to detect the needs and opportunities of an organization. Perform an analysis of the different layers or dimensions of the Enterprise Architecture: Business, Data, Applications, Technological Infrastructure, Human Resources and Integration; which are evidenced in three stages: Start, Diagnosis and Projection, each of them carries out activities aimed at improving the proper functioning of the organization. The diagnosis allows you to stop investing a lot of money and time in technology without obtaining the desired results. Through specialized methodologies such as Enterprise Architecture, problems will be solved and the opportunities present in the business will be exploited, relying on information technologies. The organization will obtain an alignment between the goals of the business and the strategies of Information Technology.

Keywords: technological diagnosis, business architecture, information technologies, strategic objectives. 


\section{INTRODUCCIÓN}

El alto nivel de complejidad de las organizaciones actuales y la necesidad imperativa de aumentar su eficacia y eficiencia en las retadoras condiciones que impone el marco económico actual representan un gran desafío para cualquier organización. Responder ágil y efectivamente a las necesidades de gestión es una tarea primordial para alcanzar el éxito en el cumplimiento de su misión. En este contexto de constante cambio y evolución surge el Diagnóstico Organizacional (DO) como muy útil y posible herramienta a utilizar.

El DO es el estudio, necesario para todas las organizaciones, que se encarga de evaluar la situación de las mismas, los problemas que existen en ellas, sus potencialidades y las vías de desarrollo para su crecimiento. En general podría decirse que busca generar eficiencia en la organización a través de cambios (Rodríguez, 2005).

EI DO, es un análisis procesal donde se examinan todas las áreas que contempla una organización para llegar a estudiarlas con profundidad y resolver situaciones que ponen en peligro el buen funcionamiento de la misma. A demás se puede definir como un proceso analítico que permite conocer la situación real de la organización en un momento dado para descubrir problemas y áreas de oportunidad, con el fin de corregir los primeros y aprovechar los segundos (Ferrer R. L., 2015).

Para la realización del diagnóstico se utiliza un enfoque de Arquitectura Empresarial (AE), que no es mas que un conjunto coherente de principios, métodos y modelos que se utilizan en el diseño y la realización a nivel empresarial de la estructura organizacional, los procesos de negocio, los sistemas de información y la infraestructura. Una $A E$ explica cómo todos los elementos de las tecnologías de la información en una organización o sea los procesos, los sistemas, la estructura organizacional y las personas se integran y trabajan de forma conjunta (Arango Serna, 2010).

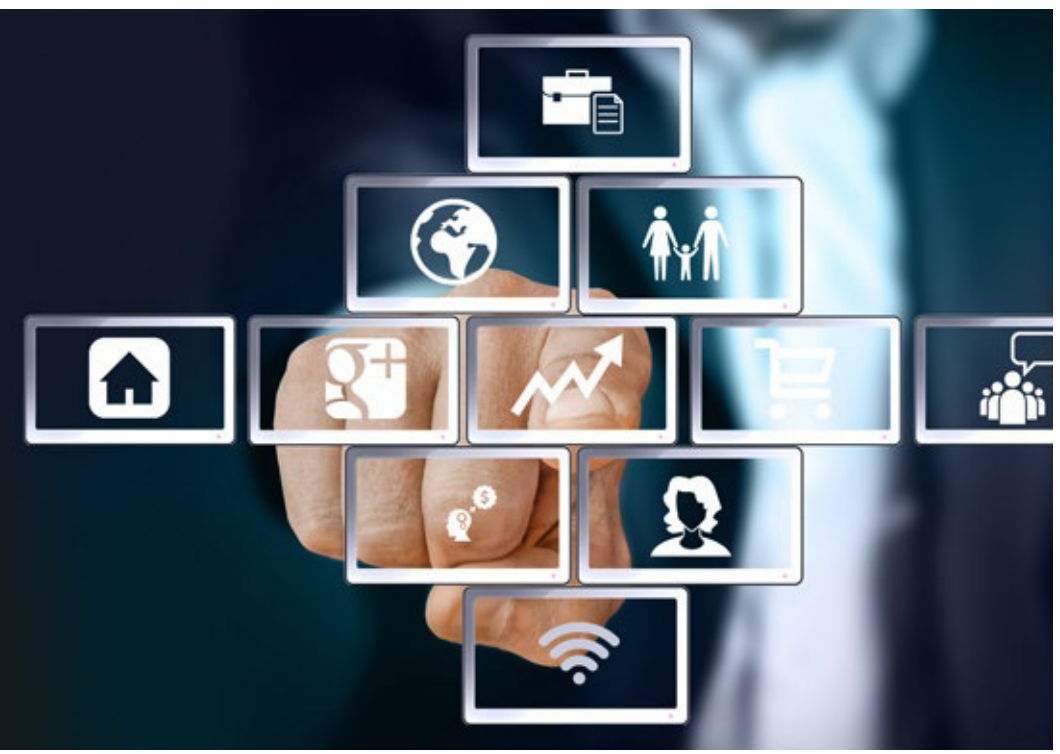

La AE constituye la línea base para cambiar la organización una vez creada, es una descripción rigurosa de la misma, la cual comprende sus componentes, las propiedades externas visibles de estos componentes y las relaciones entre ellos. Describe la terminología, la estructura de los componentes de una organización, sus relaciones con el entorno externo y los principios que guían los requerimientos, el diseño y la evolución de la misma (Ferrer R. L., 2015).

De manera general el enfoque de $\mathrm{AE}$ provee una descripción del estado actual en el que se encuentra la organización a partir de aquí proyecta un estado deseado y estima la priorización de los proyectos para transitar de un estado a otro.

Entre los múltiples factores, que afectan hoy a las organizaciones se pueden mencionar:

"Softwares viejos que no se integran coherentemente,

" Múltiples fuentes de donde buscar para tomar decisiones,

Personal de Tecnología de la Información (TI) enfocado a la solución de los problemas operacionales,

Desarrollo de la infraestructura,

- El cúmulo de documentos que dificultan la eficiencia del proceso de negocio y la demora que esto implica.

Todo lo antes expuesto provoca que la dirección de la organización no encuentra qué camino seguir para la informatización de la misma y la búsqueda de una estrategia que garantice la satisfacción de los objetivos del negocio basado en las tecnologías de la información.

La mayoría de las organizaciones no tienen claridad de lo que representa la $A E$ y de los beneficios que supone un correcto tratamiento de la información para su desarrollo futuro, se enfocan en la solución de bienes o servicios y en el cumplimiento de sus metas, descuidando o dejando en segundo plano las potencialidades que brinda la informatización para sus procesos.

Partiendo, de la situación expuesta anteriormente se plantea el siguiente problema ¿Cómo contribuir al desarrollo de las organizaciones aplicando la AE? Se desarrolla como objetivo de la investigación: Proponer la realización del Diagnóstico Organizacional utilizando el enfoque de Arquitectura Empresarial para el desarrollo de las organizaciones.

El estudio busca extender a las organizaciones las oportunidades de mejora que se tiene con la implementación de la $A E$, con la posibilidad de ser aplicable a todas las organizaciones que lo soliciten.

El contenido del artículo estará estructurado en las secciones siguientes los materiales y métodos donde se resumen los diferentes tipos de bibliografía consultada, ressultados y discusión donde se explicará la solución propuesta y se realizará una valoración de la misma, las conclusiones donde se resumen los resultados obtenidos y el aporte de ellos a la sociedad, y por último pero no menos importante las referencias que es toda la documentación consultada. 


\section{MATERIALES Y MÉTODOS}

Para la definición de los diagnósticos en tecnologías de la información se consultó bibliografía como: el Marco de Trabajo de Arquitectura del Grupo Abierto (TOGAF, por sus siglas en inglés) (The Open Group, 2013) y el marco de trabajo de arquitectura Empresarial Federal (FEAF por sus siglas en ingles) (Fernández, 2017) (Osorio, 2014) que, aunque describen un proceso, no está orientado hacia el desarrollo de un proyecto en específico, sino a la implementación de una iniciativa a largo plazo.

Otros Marcos de Trabajo consultados fueron: El marco de Trabajo de Zachman (Zachman, 2008), el Marco de Trabajo de Arquitectura del Departamento de Defensa de los Estados Unidos (DoDAF por sus siglas en inglés) (IBM Knowledge Center, 2014), o el Marco de Trabajo de Arquitectura Empresarial del Tesoro (TEAF, por sus siglas en inglés) (Tesoro Enterprise Architecture Framework, 2015), los cuales proveen un modelo de referencia que identifican y organizan los artefactos de la arquitectura, pero no describen un proceso lo suficientemente específico para cada uno de estos artefactos.

También un Marco de Negocio para el Gobierno y la Gestión de las TI en la Empresa, COBIT 5 (ISACA, 2012), el cual se construye sobre 5 principios básicos que son satisfacer las necesidades de las partes interesadas, cubrir la empresa de extremo a extremo, aplicar un marco de referencia único e integrado, hacer posible un enfoque holístico, separar el gobierno de la gestión. COBIT 5 es genérico y útil para empresas de todos los tamaños, tanto comerciales, como sin ánimo de lucro o del sector público. A demás se consultó la tesis de maestría (Ferrer R. L., 2014), donde se explica detalladamente una metodología a utilizar durante el proceso de diagnóstico TI.

Asimismo, como el vehículo para construir la estrategia de transición sería un proyecto, que, a la vez, consistiría en la prestación de un servicio tecnológico, se consultó como referente en la gestión de proyectos y la prestación de servicios tecnológicos, como los casos de: PMBOK 6 (Project Management Institute, 2017), el sitio (Universidad de Ciencias Informáticas, 2017) donde se detalla bien los procesos de mejora que debe seguir cada especialista vinculado a un proyecto siguiendo las normas establecidas por CMMI en sus niveles de madurez 2 y 3 , y el libro de CMMI para el desarrollo, versión 1.3 (CMMI, 2010), asi como la linea base de proyectos de IPMA (IPMA, 2017), el manual de PRINCE2 (Escuela de Organización Industrial, 2014).

Como resultado de este estudio, y a partir de que el mismo parte de necesidades en el Centro de Consultoría y Desarrollo de Arquitecturas Empresariales se decidió tomar como bases fundamentales para la creación del modelo a TOGAF, el cual resulta el marco de trabajo que más se asemeja a los objetivos estratégicos establecidos en el CDAE, en cuanto a la integridad de procesos, la neutralidad de proveedores y la disponibilidad de información, teniendo como punto más débil la no existencia de un modelo para medir la madurez dentro del marco, para lo cual se utilizara a PMBOK como referencia para la gestión del proyecto y CMMI-DEV como guía en los aspectos relativos a la prestación de un servicio tecnológico de calidad.

A su vez el estudio realizado se utilizó como base en la metodología a utilizar en el desarrollo de un proyecto de diagnóstico en tecnologías de la información que es el dominio de aplicación de esta investigación.

\section{RESULTADOS Y DISCUSIÓN}

Como primer resultado de la investigación en concordancia con el objetivo propuesto se encuentra la obtención de una metodología de trabajo para la proyección es-

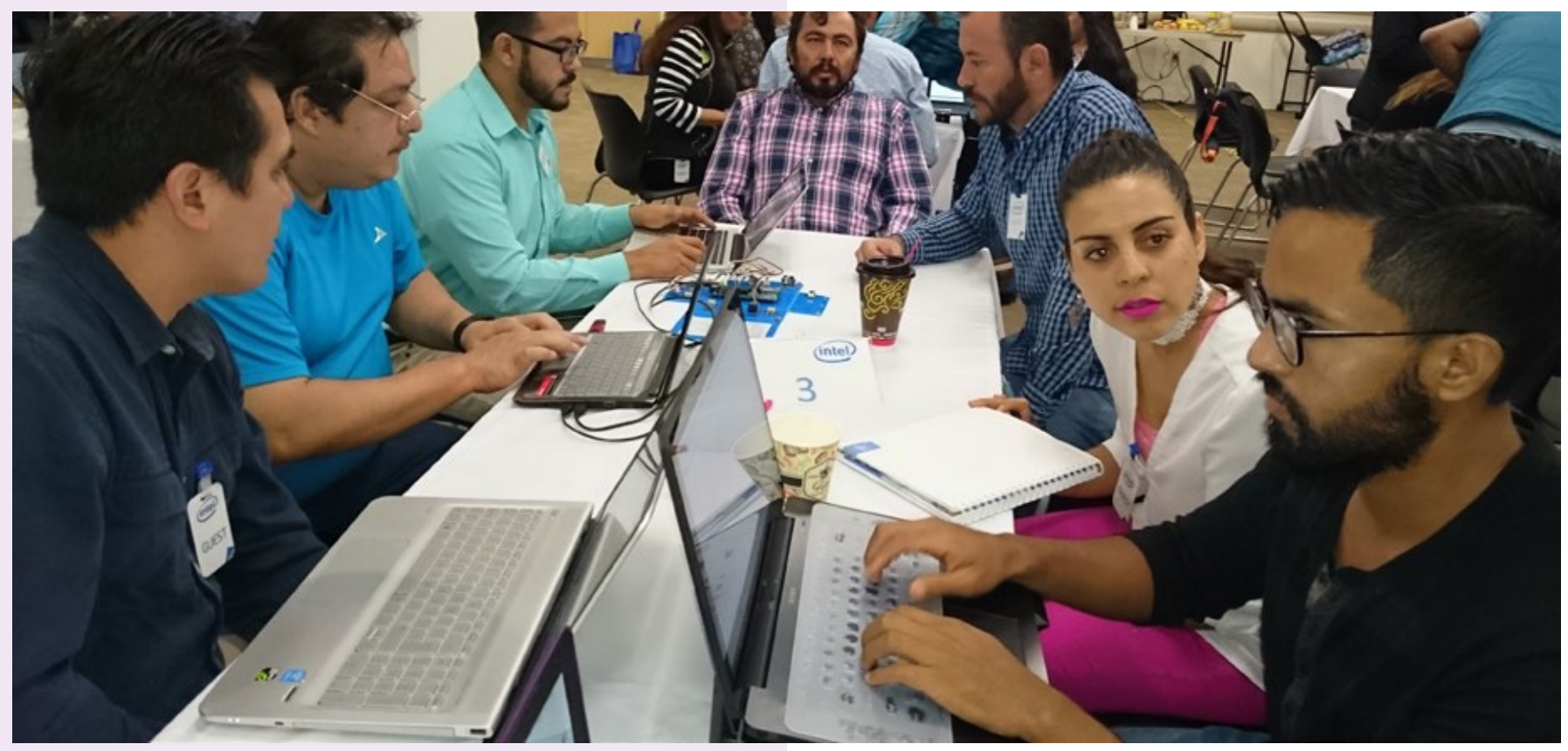




\section{Etapa de Proyección:}

Partiendo de las oportunidades de mejoras detectadas en el estado actual de la organización, se realiza una proyección estratégica la cual debe corresponder con la visión y los objetivos estratégicos planteados anteriormente. Con esta proyección se realiza un análisis de brecha, el cual servirá como base para trazar el camino a seguir durante la transición. Se definen además una serie de estados intermedios que permitirán chequear la obtención de resultados medibles en el corto y mediano plazo, que a su vez tributen a la consecución del estado deseado integral. Se realiza también la priorización de los proyectos en dependencia de las necesidades de la organización.

Siguiendo las etapas de la metodología antes explicada se aplica el servicio de Diagnóstico en Tecnologías de la Información a varias organizaciones algunas se encuentran en perfeccionamiento empresarial ${ }^{1}$.

En todos los casos de estudio se evaluaron todas las dimensiones de la $A E$, en el caso de las organizaciones en perfeccionamiento, tienen implementado el sistema de gestión de la Calidad por lo que en la mayoría de los casos tienen un enfoque vinculado a procesos siendo así más sencillo para revisar la dimensión de negocio, y proyectarnos en resto de las dimensiones, en el caso de las organizacionesque no se encuentran en perfeccionamiento algunas siguen un enfoque por procesos y otras que son la mayoría siguen un enfoque funcional o sea en función de las funciones establecidas por cargo, esto implica que la forma de trabajo sea diferente pues en un enfoque funcional cada persona trabaja de forma independiente centrados solamente en las funciones del cargo que están ejecutando, en el caso del enfoque por procesos, inciden varios departamentos, permitiendo así que el flujo de información sea rápido y veraz y que la información viaje en un solo canal, además evita la duplicidad de la misma.

Tabla 1: Características de las Empresas

\begin{tabular}{|l|l|}
\hline Tipología & \multicolumn{1}{|c|}{ Organizaciones } \\
\hline Tipo 1 & Medicina o Salud \\
\hline Tipo 2 & Empresas de la Construcción \\
\hline Tipo 3 & Centros de Investigación \\
\hline Tipo 4 & Universidades \\
\hline Tipo 5 & Empresas Productoras y Comercializadora \\
\hline
\end{tabular}

Tomaremos como ejemplo las organizaciones de Tipo 5, en la cual se aplicará el servicio de Diagnóstico en Tecnologías de la Información (DTI).

La misma como se puede evidenciar en la Tabla 1, es una empresa productora y comercializadora que, aunque se encuentra en perfeccionamiento están dando los primeros pasos por lo que no tienen bien definido el Sistema de Gestión de la Calidad.

Para la aplicación del servicio de DTI primero se evalúan todas las dimensiones de la $\mathrm{AE}$, dimensión de negocio, dimensión de datos, dimensión de aplicaciones, dimensión de infraestructura, dimensión de recursos humanos y dimensión de integración. Partiendo del estado actual en que se encuentra la organización para proyectar un estado deseado y a su vez proporcionar una priorización de los proyectos.

\section{Estrategia de transición de la AE para las Organizaciones de Tipo 5}

La estrategia de transición en correspondencia con el modelo desarrollado estará conformada por los siguientes elementos.

\section{Estado Actual}

Se realizó una definición del estado actual de la $A E$, abarcando todos los procesos de la organización (estratégicos, claves y de apoyo) haciendo especial énfasis en los procesos claves o misionales, incluyendo además una evaluación de todas las dimensiones propuestas en (Ferrer R. L., 2014) y se incluyó ademas la dimensión de integración que va a permitir corroborar la interrelación que debe existir entre todas las demás dimensiones de la arquitectura empresarial, el cual puede resumirse de la siguiente manera.

A partir de herramientas y técnicas utilizadas, se realizó una aproximación de los procesos de la organización teniendo en cuenta la distribución de los mismos, se confeccionaron varios artefactos que nos proporcionaron una vista arquitectónica de la organización como son:

$\leadsto$ Mapa de Procesos de la organización

Descripción de los procesos misionales de la organización

M Identificación del flujo de Datos

Mapa Conceptual

Descripción de las aplicaciones

M Modelación y Descripción de la red

Descripción de los recursos humanos a partir de sus competencias y capacidades

Todo ello, permitió arribar a la conclusión de que los macro-procesos definidos en este tipo de organización no son documentados, ni gestionados pues no están estandarizadas las maneras de proceder y colaborar en función de un objetivo común, los procesos no pueden ser

1 Perfeccionamiento Empresarial: Es un sistema de dirección y gestión empresarial cuyo objetivo central es incrementar al máximo la eficiencia y competitividad de la organización estatal socialista sobre la base de otorgarle las facultades y atribuciones necesarias para una correcta administración, con una concepción de avanzada en la gestión empresarial así como las políticas, principios y procedimientos que propendan al desarrollo de la iniciativa, la creatividad y la responsabilidad de todos los directivos y trabajadores. 
estudiados y mejorados en función de sus oportunidades de mejora, no se pueden gestionar los requisitos de los procesos para el desarrollo de software y por tanto no se puede rediseñar los procesos cuando se adoptan nuevas tecnologías.

Basados en un estudio realizado a la documentación, se puede evidenciar que no se realizan análisis de los datos previendo los futuros comportamientos de los mismos, sino que solamente se analiza el pasado, además no se encuentra evidencia de los análisis realizados por lo que no se estandarizan las vistas graficas de la información que permiten aplicar inteligencia en los análisis realizados para la toma de decisiones estratégicas. Además, no se muestra la integración que debe existir en la información que manejan los procesos de gestión para realizar análisis integral de la información que trascienda las áreas funcionales y la duplicidad de la información.

Poseen un nivel de automatización medio, aunque las aplicaciones con las que trabajan no se integran completamente y no se explotan al máximo algunos sistemas. A demás se previó la informatización sin una concepción del futuro por lo que se instalaron aplicaciones para resolver problemáticas actuales sin tener un enfoque previo del proceso completo lo que trajo consigo nuevas no conformidades sin resolver.

En este caso cuentan con una infraestructura de centro de datos adecuada para satisfacer sus necesidades actuales (servicios telemáticos, aplicaciones en uso, servicios desplegados). Para el caso de los recursos humanos de $\mathrm{TI}$ y el personal que trabaja con los sistemas informáticos no cuentan con las competencias suficientes para afrontar la informatización.

De forma general podemos decir que los procesos y las funciones asociadas a la mejora organizacional basadas en las capacidades de TIC requieren ser optimizados.

\section{Estado Deseado}

Sobre la base del estado actual antes descrito, se proyectó un estado deseado de la $A E$, que también abarcó todas las dimensiones propuestas en (Ferrer R. L., 2014) y además la dimensión de integración, lo que puede resumirse de la siguiente manera.

Los procesos deben ser descritos y modelados, además de optimizados y gestionados en función de sus oportunidades de mejora. Sus definiciones deben ser conocidas y comprendidas por todos los involucrados.

Asimismo, se propone la creación de modelos de datos que respondan a estándares establecidos, abarcando la captura, almacenamiento, integración y análisis de los datos que provengan tanto de fuentes automatizadas como de los provistos por el personal que labora en los procesos. A continuación, una propuesta de aplicaciones a partir de los procesos identificados, la cual se evidencia en la Tabla 2.

Tabla 2: Matriz de Aplicaciones Vs Procesos

\begin{tabular}{|c|c|c|c|c|c|c|c|c|}
\hline $\begin{array}{l}\text { Aplicaciones D } \\
\text { Procesos } \nabla\end{array}$ & $\begin{array}{c}\text { Diagnóstico } \\
\text { y Migración a } \\
\text { Software Libre }\end{array}$ & $\begin{array}{c}\text { Plataforma } \\
\text { Web } \\
\text { Integradora }\end{array}$ & $\begin{array}{l}\text { Sistema de } \\
\text { Planificación }\end{array}$ & $\begin{array}{l}\text { Sistema para } \\
\text { la Gestión } \\
\text { Documental }\end{array}$ & $\begin{array}{l}\text { Sistema para } \\
\text { la Gestión de } \\
\text { la Producción }\end{array}$ & $\begin{array}{l}\text { Sistema de } \\
\text { Contratación }\end{array}$ & $\begin{array}{l}\text { Sistema } \\
\text { Contable }\end{array}$ & $\begin{array}{l}\text { Portal } \\
\text { Web }\end{array}$ \\
\hline $\begin{array}{l}\text { Gestión Estratégica } \\
\text { Institucional }\end{array}$ & $x$ & $\mathrm{x}$ & $x$ & $\mathrm{x}$ & $x$ & $\mathrm{x}$ & $x$ & $x$ \\
\hline $\begin{array}{l}\text { Gestión de la } \\
\text { Producción }\end{array}$ & $\mathrm{x}$ & & $x$ & $x$ & $x$ & $x$ & & \\
\hline Gestión Comercial & $x$ & & $x$ & $x$ & $x$ & $x$ & & \\
\hline $\begin{array}{l}\text { Gestión de los } \\
\text { Recursos Humanos }\end{array}$ & $\mathrm{x}$ & & $x$ & $\mathrm{x}$ & $\mathrm{x}$ & $\mathrm{x}$ & $x$ & \\
\hline $\begin{array}{l}\text { Gestión Contable } \\
\text { Financiera }\end{array}$ & $\mathrm{x}$ & & $x$ & $\mathrm{x}$ & $\mathrm{x}$ & $x$ & $\mathrm{x}$ & \\
\hline $\begin{array}{l}\text { Gestión de los } \\
\text { Servicios Internos }\end{array}$ & $\mathrm{x}$ & & $\mathrm{x}$ & $\mathrm{x}$ & & & & \\
\hline $\begin{array}{l}\text { Gestión de las } \\
\text { tecnologías de la } \\
\text { información }\end{array}$ & $x$ & $\mathrm{x}$ & $x$ & $\mathrm{x}$ & $x$ & $x$ & $x$ & $x$ \\
\hline
\end{tabular}

En cuanto a infraestructura tecnológica se realizó una distribución de como quedarían sus servicios y aplicaciones virtualizados con la tecnología con que cuentan actualmente y luego se realizó un trabajo detallado de una propuesta de adquisición de equipamiento para soportar los servicios y aplicaciones que se proponen anteriormente realizar.

Por último, se proyectó la realización de un programa de capacitación especializado, partiendo de la identificación de los roles definidos en cada área. 


\section{Priorización de Proyectos}

Se realizó la priorización de los proyectos a partir de los siete sistemas identificados para la informatización de los procesos, los cuales responden a los objetivos estratégicos del negocio de la organización se pueden evidenciar en la Tabla 2. Se recomendó que como el personal de TI no cuenta con las competencias necesarias todos los proyectos fueran contratados con otras organizaciones que se dediquen a generar productos y servicios de software.

\section{Beneficios percibidos con la aplicación de un enfoque de $\mathrm{AE}$}

Una vez aplicada la Metodología para la realización del Diagnóstico en Tecnologías de la Información en la organización, se perciben beneficios entre los que se pueden destacar:

Definición de un plan estratégico de la organización que tiene en cuenta el negocio, la información, aplicaciones, infraestructura tecnológica, los recursos humanos y una manera de integrar cada uno de ellos.

Posibilidad de integrar y reutilizar aplicaciones y recursos en toda la organización.

Permite conocer el estado ideal al que podría llegar la organización y el papel de la tecnología para soportar los procesos de negocio necesarios para alcanzarlos.

- Brinda herramientas para optimizar los procesos.

\section{CONCLUSIONES}

Se explica el proceso de diagnóstico en tecnologías de la información siguiendo un enfoque de arquitectura empresarial lo que posibilita un mejor entendimiento de los beneficios que reporta para las organizaciones.

Se aplica la metodología minuciosamente, logrando resultados relevantes en cada una de las entidades objeto del diagnóstico.

Con la aplicación del diagnóstico en tecnologías de la información, utilizando un enfoque de arquitectura empresarial, se demuestra la necesidad de informatización de las organizaciones y con esto se potencia el desarrollo de las tecnologías de la información y las comunicaciones en el sector empresarial.

La puesta en práctica de estrategias y el modelado de la arquitectura empresarial puede presentar múltiples ventajas, en función del ámbito que sea analizado y la propia estructura del negocio, por lo que se puede llegar a la conclusión de que aplicando un enfoque de $\mathrm{AE}$ se obtiene:

Una disminución de los gastos y mayor rendimiento de las inversiones realizadas en tecnologías de la información.

" La consolidación de las infraestructuras de los sistemas de información.
- La documentación y estandarización de los procesos.

Preservación del conocimiento y la memoria corporativa. $\leadsto$ Unificación y mejora en la toma de decisiones empresariales, incluyendo la gestión del cambio.

$\rightarrow$ Alineamiento de las necesidades del negocio con los servicios ofrecidos por los sistemas de información. Mejora de la comunicación interna.

Automatización de procesos.

\section{BIBILIOGRAFÍA}

[1] Arango Serna, L. S. (2010). Arquitectura Empresarial. Una visión general. Revista de Ingenierías. Universidad de Medellín, 19(16), 101-111.

[2] CMMI. (2010). Mejora de los procesos para el desarrollo de mejores productos y servicios (Tercera ed.).

[3] Escuela de Organización Industrial. (2014). PRINCE2- Otra metodología para la gestión de proyectos. Obtenido de http://www.eoi.es/blogs/mcalidadon/2014/12/20/prince2otra-metodologia-para-la-gestion-de-proyectos/

[4] Fernández, P. S. (2017). Comparación de marcos de trabajo de arquitectura empresarial. La Habana, Universidad José Antonio Echeverría, CUJAE.

[5] Ferrer, R. L. (2014). Definición de procesos para gestionar alcance y comunicaciones en proyectos de diagnóstico organizacional enmarcados por la arquitectura empresarial. Tesis de Maestría, La Habana, Universidad de Ciencias Informáticas. Recuperado el 2017

[6] Ferrer, R. L. (2015). Definición de una estrategia de transición de la arquitectura empresarial en un entorno industrialbiotecnológico. $R C C l$.

[7] IBM Knowledge Center. (2014). Rational Systems Architect 11.4.2. Recuperado el 2018, de https://www.ibm.com/ support/knowledgecenter/es/SS6RBX_11.4.2/com.ibm. sa.dodaf.doc/topics/t_creadodafprds.html

[8] IPMA. (2017). IPMA, International Project Management Association.

[9] ISACA. (2012). COBIT 5, Un Marco de Negocio para el Gobierno y la Gestión de las TI en la Empresa. Reino Unido.

[10] ISO/IEC/IEEE 42010. (s.f.). Systems and software engineering.

[11] Lankhorst, M. (2013). Enterprise Architecture al work: Modelling, Communication and Analysis.

[12] Osorio, A. F. (2014). FEAF The Federal Enterprise Architecture Framework (FEA). Goverment Enterprise Architecture (GEA).

[13] Project Management Institute. (2017). A Guide to the Project Management PMBOK 6 (Sexta ed.). Recuperado el Noviembre de 2017

[14] Rodríguez, M. (2005). Diagnóstico Organizacional. México: Alfaomega Grupo editor.

[15] Tesoro Enterprise Architecture Framework. (2015). Obtenido de https://chae201511700812108.wordpress. com/2015/05/01/tesoro-enterprise-architectureframework/

[16] The Open Group. (2013). Guía de Bolsillo TOGAF 9.1. Reino Unido: Van Haren Publishing.

[17] Universidad de Ciencias Informáticas. (2017). Mejora de Procesos de Software. Recuperado el 2018, de https:// mejoras.prod.uci.cu

[18] Zachman, J. (2008). John Zachman's Concise Definition of The Zachman Framework. Zachman International Inc. Recuperado el 2017 